\title{
ATTITUDE ADJUSTMENT OF BACKFILLING SUPPORT BASED ON MECHANICAL-HYDRAULIC CO-SIMULATION
}

\author{
Meng, Z. S. ${ }^{* * *}$; Zhang, S.**; Xie, Y. Y. ${ }^{*, \# ~ \& ~ Z e n g, ~ Q . ~ L . ~}{ }^{* * * * * *}$ \\ * State Key Laboratory of Mining Disaster Prevention and Control Cofounded by Shandong Province \\ and the Ministry of Science and Technology, Shandong University of Science and Technology, \\ 266590 Qingdao, China \\ ** College of Mechanical and Electronic Engineering, Shandong University of Science and \\ Technology, 266590 Qingdao, China \\ *** College of Information Science and Engineering, Shandong Normal University, 250358 Ji'nan, \\ China \\ E-Mail: xieyunyue515@sdust.edu.cn
}

\begin{abstract}
The attitude monitoring and controlling (AMC) technology of backfilling support is critical for the development of unmanned mining. However, the underground products need to meet the explosionproof requirements, which made new AMC methods hard to be tested. To solve this problem, an AMC method was proposed, and the multi-software co-simulation technology was applied to study the automatic attitude adjustment behaviour of support. Firstly, the AMC mathematical model of the support was established. Secondly, the mechanical-hydraulic co-simulation (MHC) model was constructed using ADAMS and AMESim. The co-simulation model rationality was analysed by comparing various action sequences. Finally, by introducing an attitude calculator into the MHC model, the pose adjustment performance of the support was tested. Results show that the coupling action of the leg and equilibrium jack causes the support system vibration. The proposed AMC method can achieve the precise support attitude control. The cumulative leg action error is $\pm 0.3 \mathrm{~mm}$, while that of the equilibrium jack is $\pm 2 \mathrm{~mm}$.

(Received in May 2020, accepted in August 2020. This paper was with the authors 1 month for 2 revisions.)
\end{abstract}

Key Words: Backfilling Support, Attitude Adjustment, Vibration, Mechanical-Hydraulic Co-Simulation

\section{INTRODUCTION}

Energy is the key factor driving economic development. Although Chinese economic growth has recently slowed down, China remains the largest energy consumer in the world. In 2018, its energy consumption for $24 \%$ of global consumption. When considering energy generation sources, coal still has a leading role in Chinese energy consumption, accounting for more than $55 \%$ of the energy consumption [1,2]. Thus, underground coal automation and intelligent mining technology are critical for the development of the coal industry.

Hydraulic support is one of the fundamental tools for underground coal mining. In the underground mining process, there are two main methods of goaf management - caving method and backfilling method [3]. The latter, as a representative of green mining, is a dominant development direction of the coal industry. It reduces the damage to the mining area environment and can effectively prevent the dynamic roof disaster [4, 5]. Compared with the conventional hydraulic support, in addition to roof supporting task, the backfilling support has a unique tamping process. The backfilling support tamping process increases the support mechanism redundancy, and reduces the working face advancing efficiency; therefore, its application is limited. Additionally, the backfilling support working attitude also affects its supporting performance $[6,7]$.

By studying the attitude monitoring and controlling (AMC) technology of backfilling support, it is possible to ensure that the support rapidly fits the roof; therefore, significantly 
improving its action efficiency and automation level. Moreover, such studies can also benefit the absolute positioning of mining equipment, improve the mining equipment operation information transparency.

\section{STATE OF THE ART}

Many scholars have studied the AMC technology of hydraulic support, attempting to improve the support attitude predictability and controllability. Ge et al. and Xie et al. [8,9] developed a virtual-reality attitude adjustment model of four-leg hydraulic support by using Unity 3D. By analysing the relationship between the virtual roof and canopy, the action information required for the support can be calculated. Zhang et al., Yang et al. and Wang et al. [10-12] proposed similar relative attitude monitoring methods using a hydraulic reference support as a benchmark, the relative position and attitude were measured by placing the lidar testing device between the adjacent supports. Based on the attitude information between the adjacent supports, the absolute support group attitude is found inversely. However, due to many working face supports, the relative attitude monitoring produces a significant cumulative error. Thus, Liang et al. [13] proposed an absolute attitude monitoring method based on optical fibre sensing technology. The real-time canopy attitude monitoring was realised by arranging a fibre Bragg grating tilt sensor on the support canopy. Furthermore, Cui and Asl $[14,15]$ proposed position tracking method for multi-bar mechanism, by placing sensors on the mechanism and combining them with a Kalman filtering method, the stable output of the mechanism position was achieved.

In recent years, the rapid development of computer technology led to a wide application of co-simulation technology for solving multi-domain problems. Mezyk et al. proposed a discrete electromechanical co-simulation (EMC) method for continuous mining machine [16]. Firstly, the authors established the continuous miner cutting model with LS-DYNA. The obtained cutting loads were applied to the electrical drive model, allowing the analysis of continuous miner non-linear vibration behaviour. In papers [17-20] the researchers adopted real-time EMC methods to study robot motion control problems. They used ADAMS to build the mechanical part and MATLAB to establish the robot electrical drive controller, allowing them to realise the precise motion control of manipulator/robot. Yang et al. and Liu et al. $[21,22]$ proposed a real-time mechanical-hydraulic co-simulation (MHC) method for hydraulic pumps. The pump mechanical transmission system was established using ADAMS, while the hydraulic drive system was developed using AMESim. The hydraulic pump flow pulsation characteristics were both analysed and optimised.

The above-referenced studies indicate that the existing AMC technology has mostly adopted both theoretical analyses and experimental tests. However, the attitude variables and attitude decision variables were not clearly defined; therefore, the existing methods cannot effectively guide the support attitude reverse control. At the same time, industrial support tests are very expensive due to the environmental, economic, and other factors. Thus, it is necessary to develop a co-simulation platform for support. In the existing research, practically MHC methods were reported, while none of them refers to hydraulic support.

Therefore, in this study, the attitude variables and support decision variables were analysed from the mechanism viewpoint. Additionally, the AMC model for backfilling support was established. The MHC support model was developed by using ADAMS, AMESim. By comparing various support action sequences, the rationality and vibration characteristics of the MHC model were analysed. Finally, an attitude calculator was built based on the AMC mathematical model. By introducing the calculator into the MHC model, the performance of the AMC method was tested. This study provides novel solutions for both the automation and intelligent control technology of backfilling support attitude. 
The remainder of this study is organised as follows. A theoretical analysis of the AMC for the support is presented in Section 3. The MHC support model was established in the same section, as well as three MHC simulation schemes. The MHC model dynamic response analyses carried out under different schemes and the AMC method performance tests are presented in Section 4. Finally, summary and conclusions are included in Section 5.

\section{METHODOLOGY}

\subsection{Theoretical analysis of the AMC for backfilling support}

In this study, a ZC 7000/20/40 type backfilling support was taken as an example (the skeleton model is shown in Fig. 1). Parts designated from 1 to 7 (on the left side) belong to the front canopy side (FCS), while the parts between 5 and 11 (right) belong to the rear canopy side (RCS). The DOF of both support sides can be found using Eq. (1).

$$
f_{s}=(6-\lambda)\left(n-g-1+\sum_{i=1}^{g} f_{i}\right)
$$

where, $f_{s}$ is the mechanism DOF, $\lambda$ is the constraint, $f_{i}$ is the DOF of the $i^{\text {th }}$ kinematic pair, $n$ and $g$ are numbers of structural members and kinematic pairs, respectively.

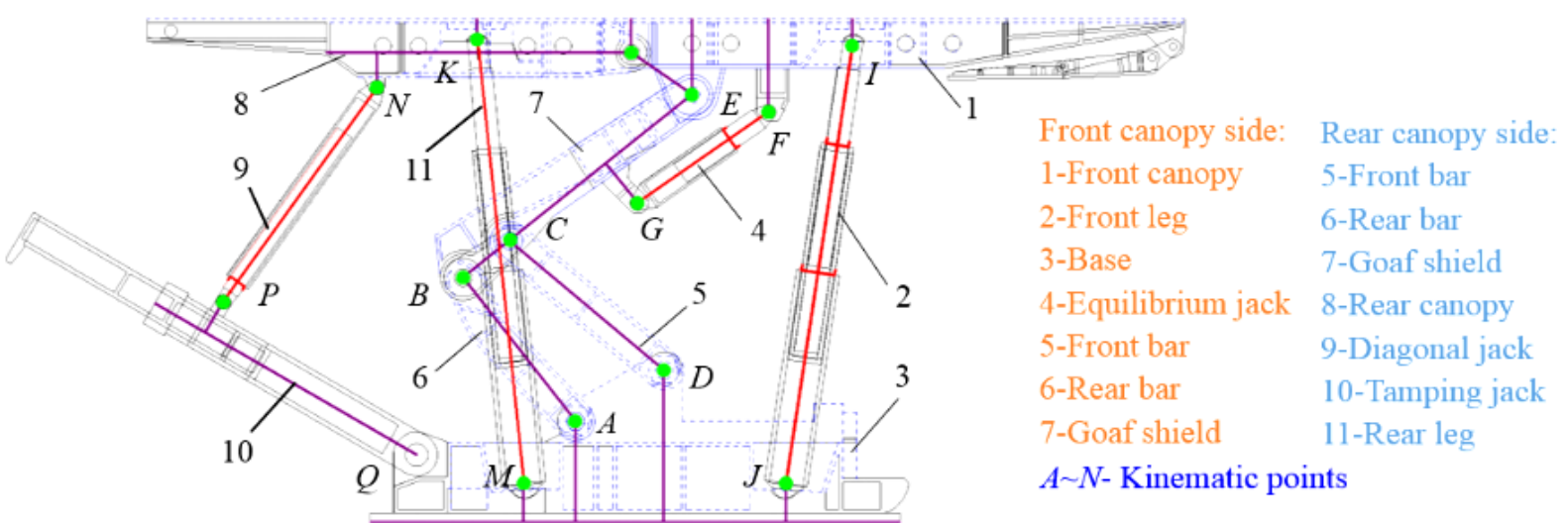

Figure 1: Plane projection model of backfilling support.

Therefore, the FCS and RCS support degrees of freedom can be expressed using Eqs. (2) and (3), respectively.

$$
\begin{gathered}
f_{s}=(6-\lambda)\left(n-g-1+\sum_{i=1}^{g} f_{i}\right)=(6-3)(10-12-1)+11=2 \\
f_{s}=(6-\lambda)\left(n-g-1+\sum_{i=1}^{g} f_{i}\right)=(6-3)(11-13-1)+12=3
\end{gathered}
$$

Once the FCS attitude is determined, the RCS degrees of freedom will be reduced to 2 . Therefore, in order to realise the support AMC, it is desirable to define the front canopy angle $(\varepsilon)$ and the rear bar angle $\left(\theta_{0}\right)$ as the attitude variables, while the leg length $\left(l_{z}\right)$ and equilibrium jack $\left(l_{q}\right)$ are chosen as the attitude decision variables. On the RCS, the rear canopy angle $\left(\varepsilon_{2}\right)$ and tamping jack $\left(\theta_{h}\right)$ are attitude variables, while the rear leg length $\left(l_{z 2}\right)$ and diagonal jack $\left(l_{t}\right)$ are as attitude decision variables. The parametric support model is shown in Fig. 2. $l_{1} \sim l_{14}$ is the length of each support part, and $\theta_{1} \sim \theta_{14}$ are the angles between said parts. Both are either known or intermediate variables. In the attitude monitoring phase, $l_{z}, l_{q}, l_{z 2}$ and $l_{t}$ are known, while $\varepsilon, \theta_{0}, \varepsilon_{2}$ and $\theta_{h}$ are unknown variables.

According to the backfilling support deformation characteristics, the FCS attitude monitoring equation is expressed as Eq. (4). 


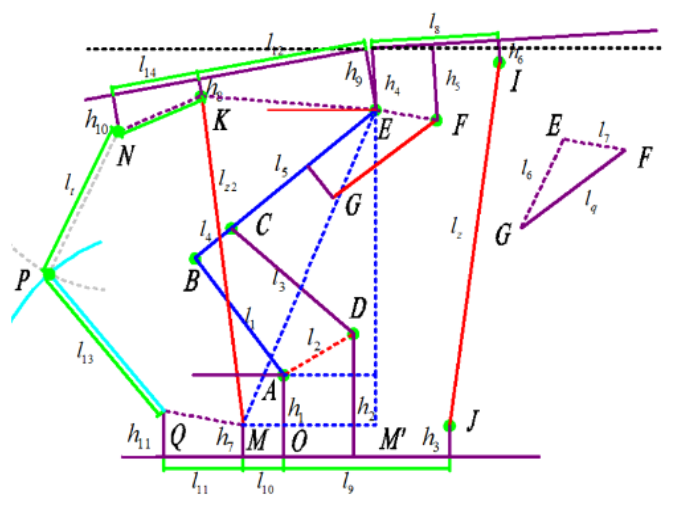

a) Length variables

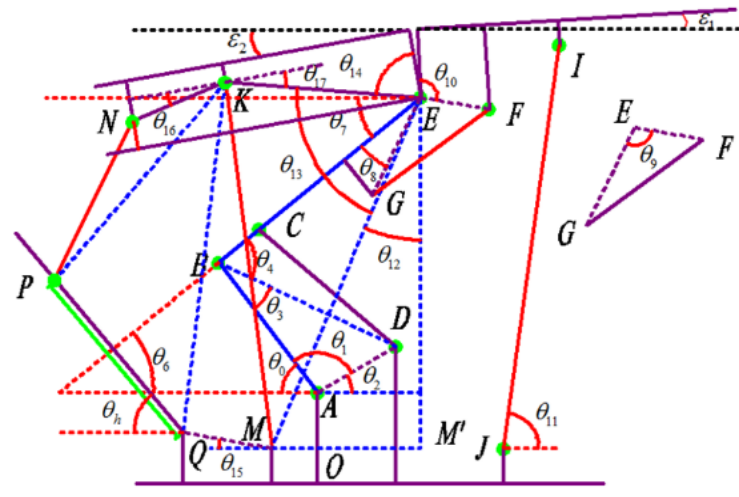

b) Angle variables

Figure 2: Parametric model of backfilling support.

$$
\left\{\begin{array}{l}
l_{z}=l_{z x}^{2}+l_{z y}^{2} \\
l_{z x}=l_{9}+l_{1} \cos \theta_{0}-l_{5} \cos \theta_{7}+h_{4} \sin \varepsilon-l_{8} \cos \varepsilon+h_{6} \sin \varepsilon \\
l_{z y}=h_{1}+l_{1} \sin \theta_{0}+l_{5} \sin \theta_{7}+h_{4} \cos \varepsilon+l_{8} \sin \varepsilon-h_{3}-h_{6} \cos \varepsilon \\
\varepsilon=\theta_{7}+\theta_{8}+\cos ^{-1}\left[\left(l_{6}^{2}+l_{7}^{2}-l_{q}^{2}\right) / 2 l_{6} l_{7}\right]+\theta_{10}-3 \pi / 2-\pi / 12 \leq \varepsilon \leq \pi / 12
\end{array}\right.
$$

where, $l_{z x}$ and $l_{z y}$ are the horizontal and vertical leg length components, respectively. Eq. (4) is a system of non-linear equations, meaning that its numerical solution can be found using the interpolation method [23]. On the RCS, there is no evident restraint between the rear leg and diagonal jack. Once the FCS attitude is determined, the RCS will degenerate into a serial mechanism. Since the tamping mechanism drive chain in RCS is very long, the coordinate method was adopted to solve $\theta_{h}$. Finally, the RCS attitude monitoring equation is expressed as follows.

$$
\left\{\begin{array}{l}
\varepsilon_{2}=\pi-\theta_{12}-\theta_{13}-\theta_{14}-\pi / 6<\varepsilon_{2}<\pi / 6 \\
\theta_{h}=\cos ^{-1}\left[\left(P_{y}-Q_{y}\right) /\left(P_{x}-Q_{x}\right)\right]
\end{array}\right.
$$

where, $P_{x}, P_{y}, Q_{x}$ and $Q_{y}$ are the point $P$ and point $Q$ coordinates. After the target FCS attitude is determined, the target length of each support driving cylinder can be predicted. This enables the cylinders to rapidly adjust the FCS to fit the roof precisely; therefore, it is possible to realise the rapid support of the surrounding rock. The FCS attitude control model is expressed as follows.

$$
\left\{\begin{array}{l}
l_{q}=l_{6}^{2}+l_{7}^{2}-\operatorname{sqrt}\left\{\cos \left[2 l_{6} l_{7}\left(\varepsilon+3 \pi / 2-\theta_{7}-\theta_{8}-\theta_{10}\right)\right] \quad l_{q}>0\right. \\
l_{z}=\operatorname{sqrt}\left(l_{z x}^{2}+l_{z y}^{2}\right) \quad l_{z}>0
\end{array}\right.
$$

Similarly, the RCS attitude control equation is established using the coordinate method.

$$
\left\{\begin{array}{l}
l_{z 2}=\operatorname{sqrt}\left(l_{E K}^{2}+l_{E M}^{2}-2 l_{E K} l_{E M} \cos \theta_{13}\right) \\
l_{t}=\operatorname{sqrt}\left[\left(P_{y}-Q_{y}\right)^{2}+\left(P_{x}-Q_{x}\right)^{2}\right]
\end{array}\right.
$$

\subsection{Preparing the co-simulation platform}

The real-time support co-simulation platform is constructed by combining ADAMS, AMESim, and MATLAB. Data transmission (force, displacement, and velocity) and cosimulation of the model is implemented using MATLAB. Since the support load is relatively low, all support parts were considered as rigid to accelerate the simulation speed [24, 25]. Furthermore, since there was no evident constraint relationship between the RCS cylinders, 
only the FCS numerical model was established. The resulting support mechanical model developed using ADAMS is shown in Fig. 3. The base was defined as fixed, all support cylinders were defined as sliding pairs, and remaining parts were defined by using the rotating pair. Additionally, marker points were established to define the interaction variables, as shown in Table I.

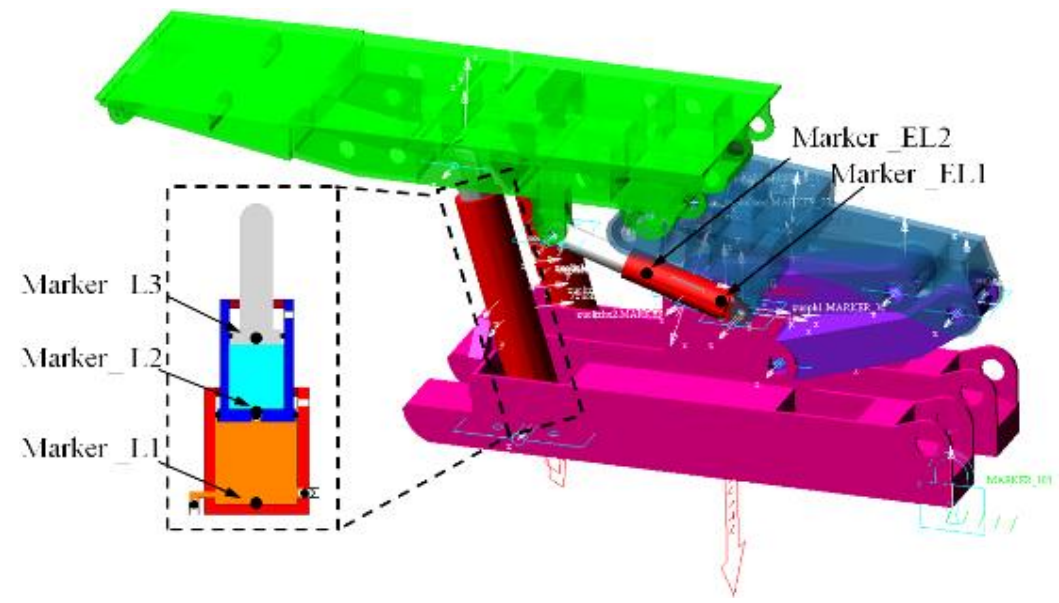

Figure 3: The FCS mechanical model of backfilling support (ADAMS).

Table I: Mechanical sub-system of backfill hydraulic support variables.

\begin{tabular}{|c|c|c|c|c|}
\hline \multicolumn{2}{|c|}{ Variable definition } & \multicolumn{2}{|c|}{ Output variable } & Input variable \\
\cline { 3 - 5 } & Displacement & Velocity & Force \\
\hline \multirow{2}{*}{ Left leg } & First stage & L_D1 & L_V1 & L_F1 \\
\cline { 2 - 5 } & Second stage & L_D2 & L_V2 & L_F2 \\
\hline \multirow{2}{*}{ Right leg } & First stage & R_D1 & R_V1 & R_F1 \\
\cline { 2 - 5 } & Second stage & R_D2 & R_V2 & R_F2 \\
\hline \multicolumn{2}{|r|}{ Left equilibrium jack } & E_LD & E_LV & E_LF \\
\hline \multicolumn{2}{|c|}{ Right equilibrium jack } & E_RD & E_RV & E_RF \\
\hline
\end{tabular}

The principle diagram of the FCS support hydraulic system is shown in Fig. 4 a. The relief valves FAD 250/50 and FAD 100/50 are used to protect both the leg and equilibrium jack. The pilot check valve FDY 400/50 and hydraulic two-way lock TMF - DSP (80/50)A are used to limit the oil flow direction. L_CS, R_CS, EL_CS, and ER_CS represent the control signal of left leg, right leg, left equilibrium jack, and right equilibrium jack directional valves. The hydraulic simulation model established in AMESim is shown in Fig. 4 b.

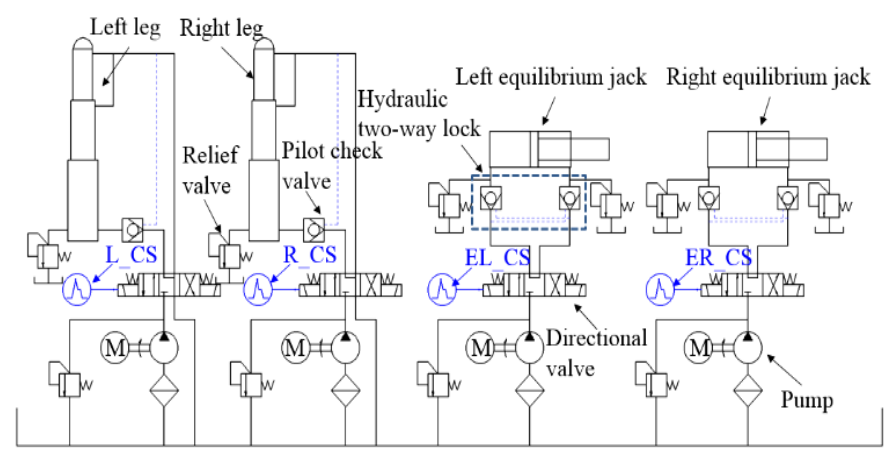

a) Hydraulic system principle diagram

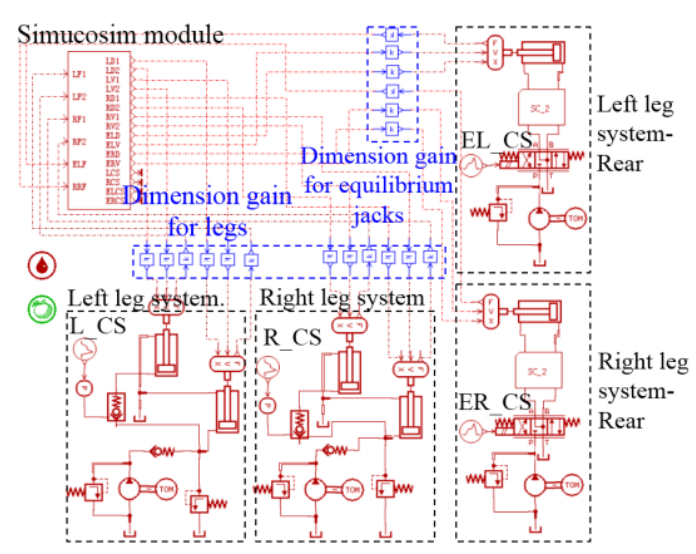

b) Hydraulic simulation model in AMESim

Figure 4: Hydraulic system principle diagram of the FCS. 


\subsection{MHC model for the backfilling support}

Based on the mechanical and hydraulic system models outlined in Section 3.2, the MHC model was established (shown in Fig. 5). The AMESim solver adopted the regular EulerLagrange method, with calculated tolerance of 1e-5. The variable step-size function ode 45 was selected as the solver (MATLAB). Data interaction interval between the three software was $0.005 \mathrm{~s}$. Meanwhile, three different action sequences were developed to evaluate the MHC co-simulation platform feasibility (Fig. 6).

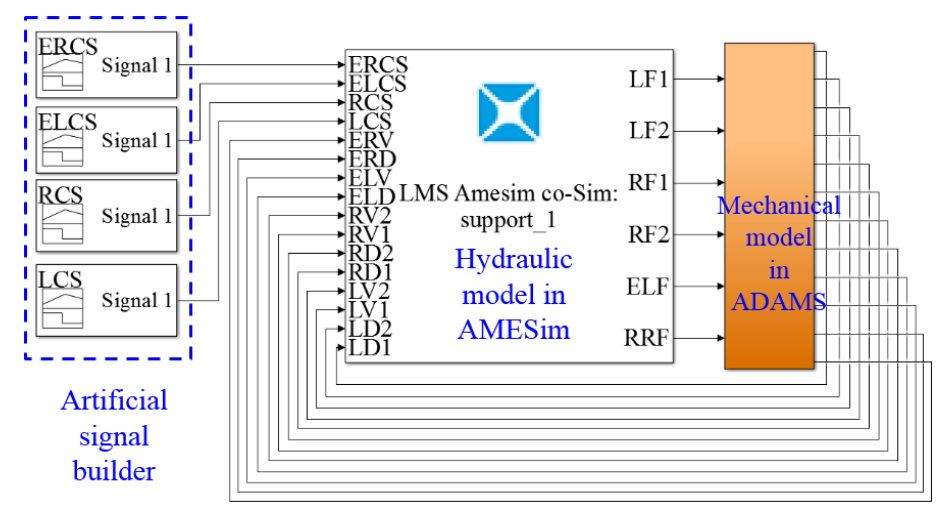

Figure 5: MHC model for backfilling support.

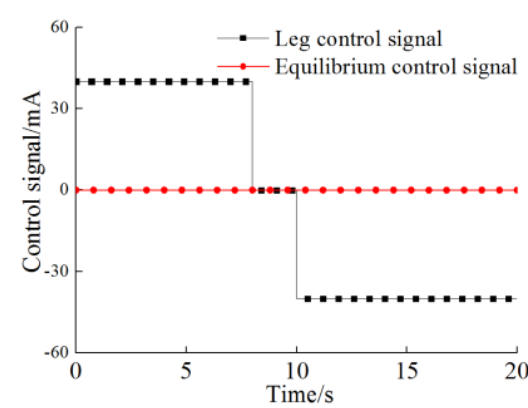

a) Scheme 1

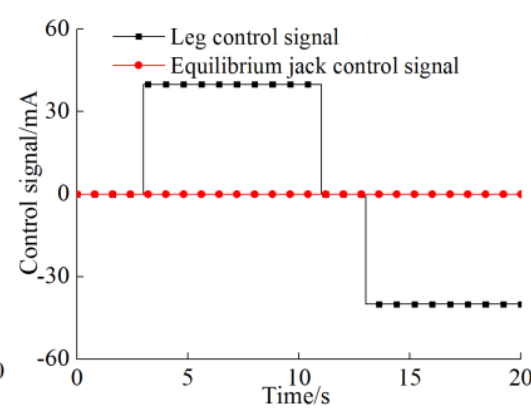

b) Scheme 2

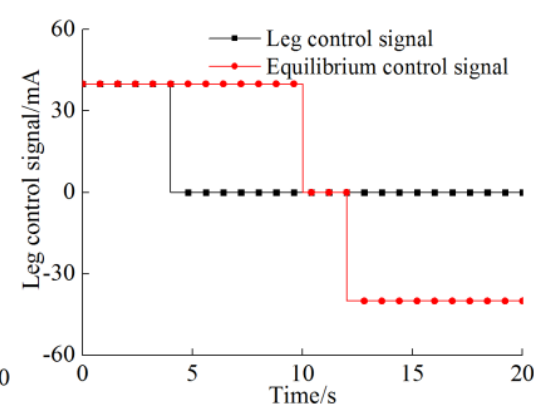

c) Scheme 3

Figure 6: The leg and equilibrium jack valve control signal.

\subsection{AMC performance test model}

An AMC calculator was established according to Eq. (6), and then brought into MHC model to replace the artificial signal builder to obtain the AMC performance test model (as shown in Fig. 7). When the upper computer inputs the support attitude signal, the calculator will provide the target lengths of driving cylinders, and then compares it with the current ADAMS output attitude, and then exports the valve control signal to adjust the support attitude.

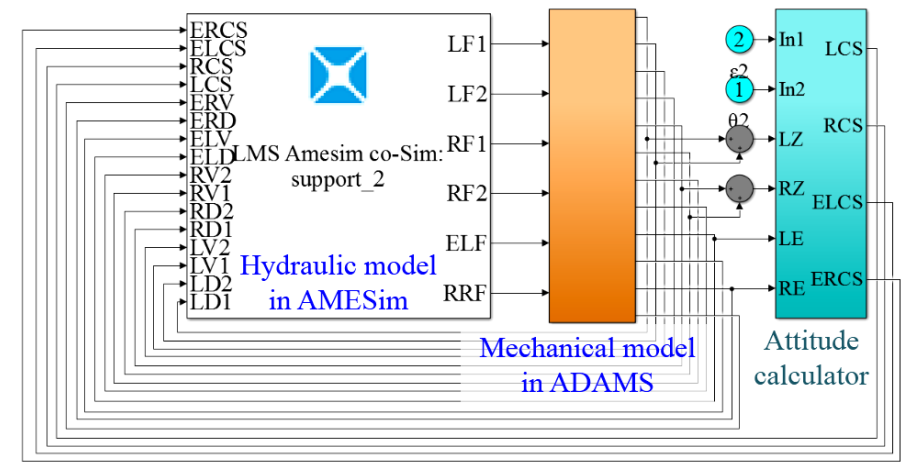

Figure 7: AMC performance test model based on MHC model. 


\section{RESULT ANALYSIS AND DISCUSSION}

\subsection{Result analysis of scheme 1 for MHC model}

Fig. 8 shows the dynamic response of the mechanical and hydraulic support systems for scheme 1 signal input. Complete support lifting process can be divided into three phases. The first phase includes the initial lifting $(0 \mathrm{~s}$ to $6.3 \mathrm{~s})$; at $0 \mathrm{~s}$, the first leg stage starts moving under the oil action. The stable action pressure is $0.88 \mathrm{MPa}(34514 \mathrm{~N})$. After the first stage cylinder reaches the stroke end $(970 \mathrm{~mm})$, the pressure in the first stage cylinder increases until the bottom valve is opened (5.5 MPa). Finally, the first and second stage cylinder pressures stabilise at $9.14 \mathrm{MPa}$ and $2.99 \mathrm{MPa}$, respectively. This includes both the bottom valve cracking pressure of $5.5 \mathrm{MPa}$ and valve flow pressure loss of $0.6 \mathrm{MPa}$. When the second cylinder reaches the stroke, the support enters the load-bearing stage. This stage lasts until the first stage cylinder pressure reaches $37.38 \mathrm{MPa}$. After $8 \mathrm{~s}$, the directional valve is switched to the middle position and the support disconnected from the pump. Although the directional equilibrium jack valve is locked at this moment, its pressure continually changes due to the leg action influence - the resultant force is mainly stable at $5205 \mathrm{~N}$. At $10 \mathrm{~s}$, the directional leg valves open in reverse and the support enter the unloading stage. Due to the flow capacity limitation of the pilot check valve, despite the rodless chamber output flowrate reaching $1035.7 \mathrm{~L} / \mathrm{min}$ and the return pressure of up to $37.6 \mathrm{MPa}$, the rod chamber inlet flowrate is only $90 \mathrm{~L} / \mathrm{min}$. Thus, it is necessary to include a special return valve to achieve rapid support unloading.

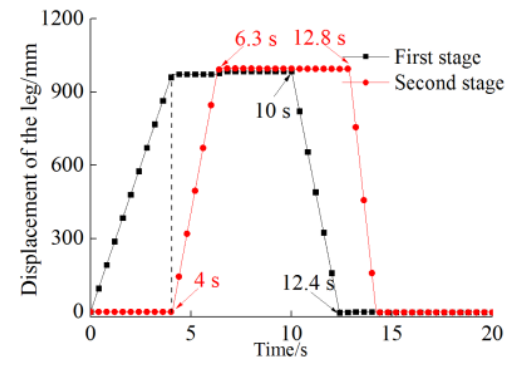

a) Leg displacement response

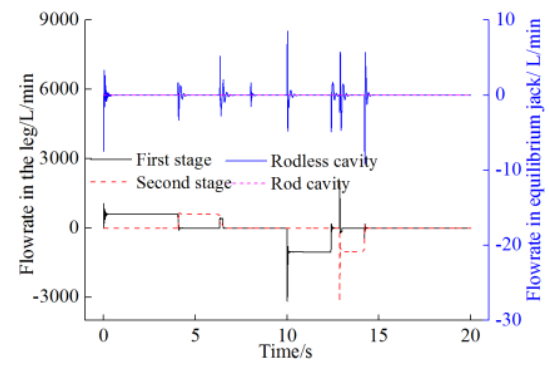

d) Leg and equilibrium jack force responses

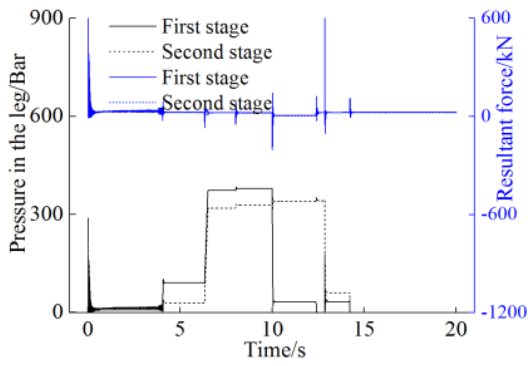

b) Leg pressure response

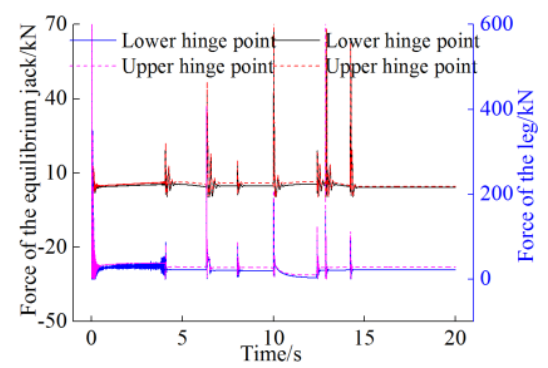

e) Leg hinge point force responses

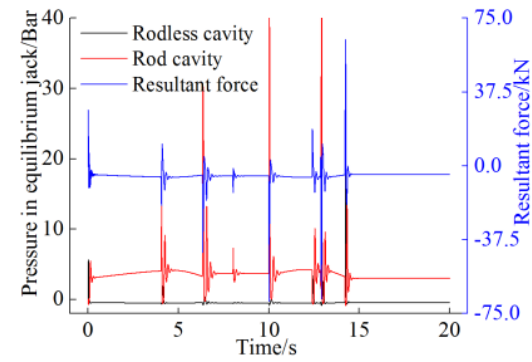

c) Equilibrium jack pressure response

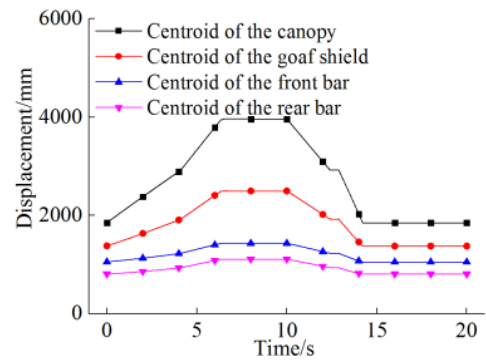

f) Displacement responses of structural parts

Figure 8: Dynamic response results of support mechanical and hydraulic systems.

\subsection{Result analysis of scheme 2 for MHC model}

In scheme 1, the support is not going through the initial gravity balance stage. When compared with scheme 1 , scheme 2 includes the initial gravity balance stage $(3 \mathrm{~s})$. The displacement and pressure response results of the leg and equilibrium jack are compared (see Fig. 9). It is apparent that in the initial gravity balance stage, there is no support oil input, meaning that the leg system has a more considerable vibration. After the initial gravity balance stage, the pressure response fluctuation of the leg and equilibrium jack in scheme 2 is 
smaller than scheme 1. However, once the system is stable, the support leg system and the equilibrium jack system responses show good convergence consistency, proving that the proposed MHC method has good reproducibility.

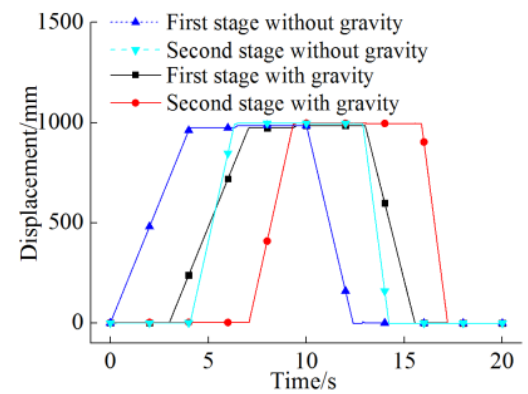

a) Leg displacement

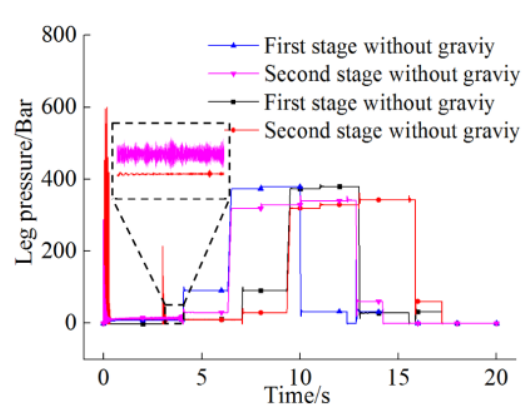

b) Leg pressure

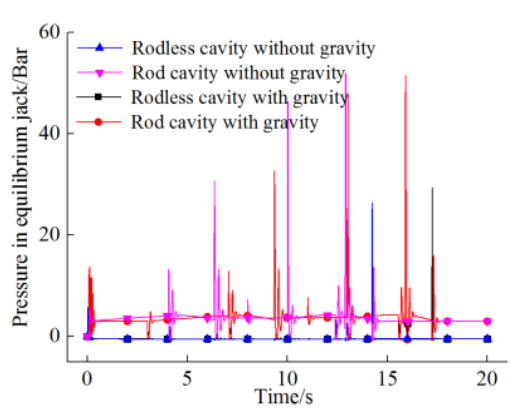

c) Equilibrium jack pressure

Figure 9: Result comparison between scheme 2 and scheme 1.

\subsection{Result analysis of scheme 3 for MHC model}

Scheme 3 aimed to test the action fluency of the whole support system. The rated oil supply of the equilibrium jack pump is $15 \mathrm{~L} / \mathrm{min}$. The pilot check valve flow rate is $50 \mathrm{~L} / \mathrm{min}$. The support system dynamic response under the scheme 3 is shown in Fig. 10. As shown, leg displacement is mainly consistent with scheme 1 during the first 4 seconds. Then, the directional leg valve switches to the middle position. During this period, the equilibrium jack can act freely independently of the leg movement.

It is worth noting that the system vibrations cannot be ignored when the oil is pumped into the rodless chamber of the equilibrium jack ( $12 \mathrm{~s}$ to $20 \mathrm{~s})$. After analysing the pressure changes of the leg and equilibrium jack, the support vibration originates from the equilibrium jack at $10 \mathrm{~s}$. By debugging the equilibrium jack using AMESim, the authors found that the vibration is caused by the frequent opening and closing of the two-way hydraulic lock. Fig. 11 illustrates the response results after increasing the flow pressure of the hydraulic lock. It can be noted that after increasing the flow pressure, the vibration phenomenon is effectively filtered out. Due to the load on the equilibrium jack is minimal $(5000 \mathrm{kN})$, therefore, the equilibrium jack cannot establish the effective working pressure. When the gravity load and potential inertial energy are introduced, the equilibrium jack system displays strong vibration. By increasing the hydraulic lock flow pressure, the pressure between the input and output ends of the hydraulic lock will be more stable.

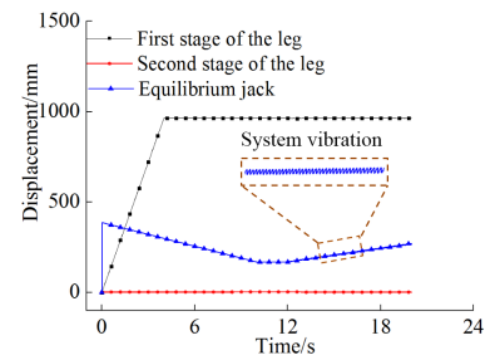

a) Displacement response

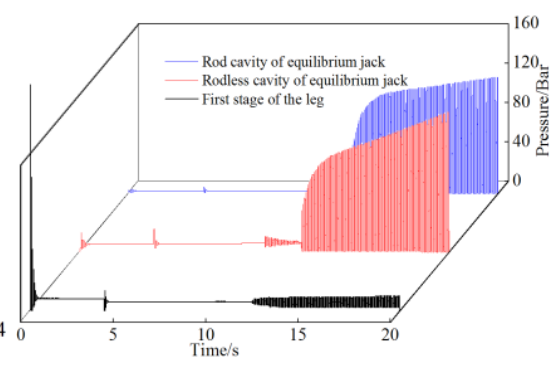

b) Pressure response

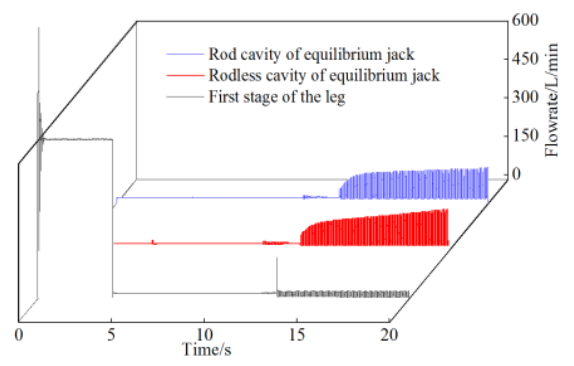

c) Flowrate response

Figure 10: Comparison of results between scheme 2 and scheme 1.

\subsection{Result analysis for AMC performance test model}

In section 4.3, the rationality of the MHC platform is analysed. In this section, the attitude calculator is introduced to the MHC platform to adjust the support attitude. Fig. 12 shows the target length of the leg and equilibrium jack calculated by the attitude calculator after 
inputting $\theta_{0}$ and $\varepsilon$. The control signal response of directional valves and displacement response results of the leg and equilibrium jack are shown in Fig. 13.

As can be seen, at $0 \mathrm{~s}, l_{z}$ is equal to $1212 \mathrm{~mm}$, and $l_{q}$ is $1625 \mathrm{~mm}$, which has a significant gap from the target attitude. The calculator outputs the maximum control signal (40 mA). By observing the directional valve action efficiency, it is clear that due to the higher quality and higher working pressure of the leg, a small amplitude vibration appears once the leg reaches the target attitude ( $4.9 \mathrm{~s}$ and $6.9 \mathrm{~s}$ ). Then, the accuracies of leg and equilibrium jack were observed. The expected leg output lengths are $2939 \mathrm{~mm}$ and $3100 \mathrm{~mm}$ at $6.5 \mathrm{~s}$ and $15 \mathrm{~s}$, respectively, while the actual lengths are $2938.7 \mathrm{~mm}$ and $3100.3 \mathrm{~mm}$. At $5 \mathrm{~s}, 8 \mathrm{~s}$, and $15 \mathrm{~s}$, the expected output lengths of the equilibrium jack are $1055.25 \mathrm{~mm}, 950 \mathrm{~mm}$, and $1100 \mathrm{~mm}$ (corresponding measured lengths are $228.6 \mathrm{~mm}, 123.35 \mathrm{~mm}$, and $273.35 \mathrm{~mm}$ ), while the actual equilibrium jack lengths are $230.58 \mathrm{~mm}, 125.17 \mathrm{~mm}$, and $271.51 \mathrm{~mm}$. During the whole period, the cumulative average leg error is $\pm 0.3 \mathrm{~mm}$, with action error less than $0.03 \%$ and calculation error is less than $0.01 \%$. The cumulative average equilibrium jack error is $\pm 2 \mathrm{~mm}$, with action error less than $1.6 \%$ and calculation error less than $0.21 \%$. Thus, the proposed AMC method and MHC system have a high calculation and control accuracies.

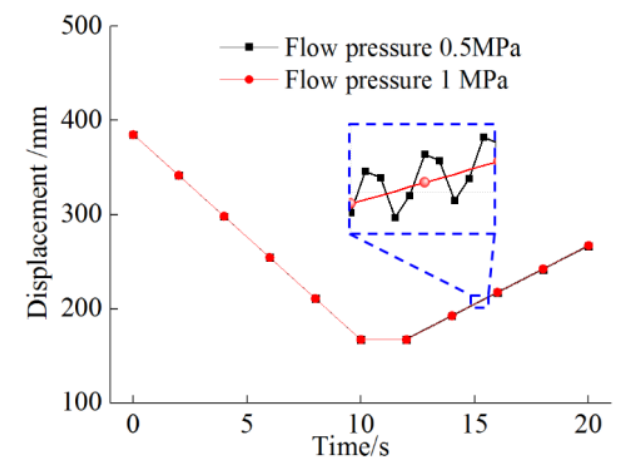

Figure 11: Equilibrium jack system response after increasing the flow pressure.

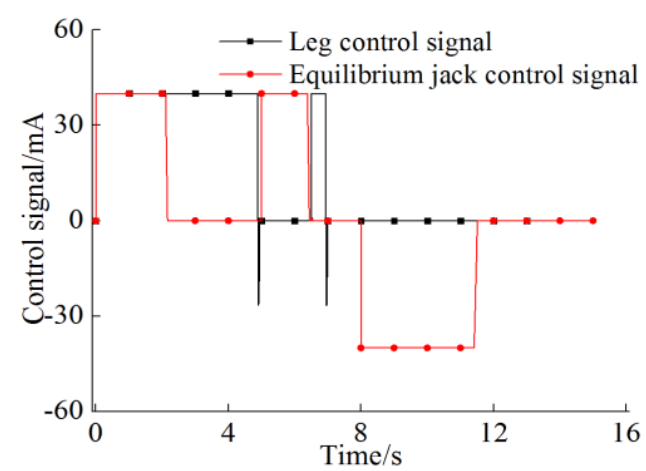

a) Control signal response of the valves

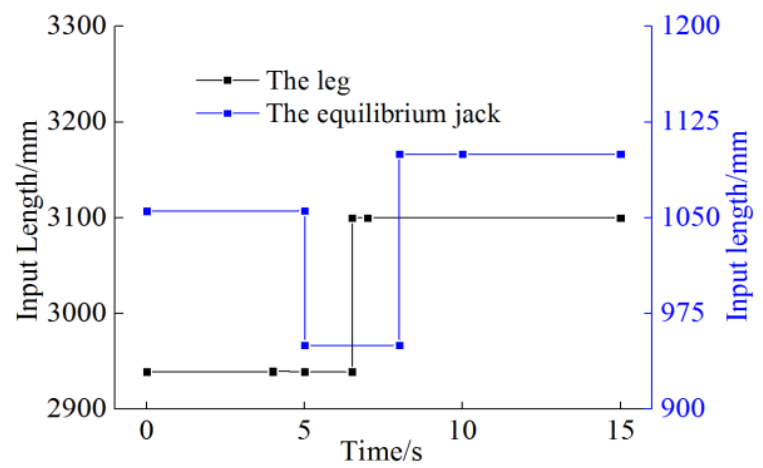

Figure 12: Digital engineering for productivity improvement.

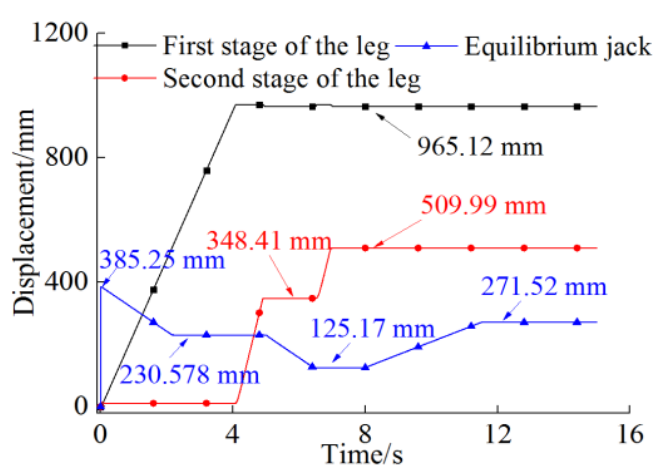

b) Displacement response of the cylinders

Figure 13: Response of the AMC performance test model.

\section{CONCLUSION}

The method for AMC analysis was proposed based on the investigation of backfilling support deformation characteristics in the lifting process. Additionally, the MHC platform was built based on multi-software co-simulation. The AMC feasibility and the factors influencing the system vibration were analysed and discussed. Based on the findings, the following conclusions were drawn:

(1) Based on AMESim, ADAMS, and MATLAB, the MHC support platform was built. By varying reference groups, support operation characteristics considering gravity load, non- 
gravity load, and simultaneous action of both the leg and equilibrium jack were analysed and discussed. The results show that the MHC platform is reliable and has good reproducibility. The simulation results describe the real support action well, thus laying a good foundation for the AMC performance test.

(2) The action coupling effect of the leg and equilibrium jack has a negative impact on the support stability. Moreover, due to the smaller cylinder diameter, the equilibrium jack is characterised by higher pressure and flow vibration. By increasing the flow pressure or setting a back pressure on the two-way hydraulic lock, the equilibrium jack system can establish a higher initial attitude adjustment pressure; therefore, reducing the vibration.

(3) During the whole attitude adjustment period, the cumulative leg action error is $\pm 0.3 \mathrm{~mm}$, equating to the leg error rate below $0.03 \%$. In equilibrium jack, cumulative action error is $\pm 2 \mathrm{~mm}$, with an error rate below $0.16 \%$. Therefore, the proposed AMC method can achieve precise support attitude control.

An MHC model of backfilling support attitude adjustment is established in this study, and the AMC method rationality is tested. However, hydraulic support is a complex assembly. The change in the hinge joint clearance will also affect the support position, which was not considered in this study. Thus, in the future, the influence of hinge point clearance should be considered, aiming to establish a more comprehensive support attitude adjustment model. Finally, the fusion of attitude information between supports should be considered.

\section{ACKNOWLEDGEMENT}

This work was supported in part by the Key Research and Development project of China (Grant No. 2017YFC0603005), Natural Science Foundation of China (Grant Nos. 51704178 and 51974170), and the Natural Science Foundation of Shandong Province (Grant No. ZR2019MEE067).

\section{REFERENCES}

[1] BP Corporation. BP Energy Outlook 2019, from https://www.bp.com/en/global/corporate/energyeconomics/energy-outlook.html, accessed on 18-06-2019

[2] Wu, Q. S.; Jiang, L. S.; Wu, Q. L.; Xue, Y. C.; Gong, B. (2018). A study on the law of overlying strata migration and separation space evolution under hard and thick strata in underground coal mining by similar simulation, DYNA, Vol. 93, No. 2, 175-181, doi: $10.6036 / 8678$

[3] Tai, Y.; Han, X. L.; Huang, P.; An, B. F. (2019). The mining pressure in mixed workface using a gangue backfilling and caving method, Journal of Geophysics and Engineering, Vol. 16, No. 1, 1-15, doi:10.1093/jge/gxy001

[4] Zhang, Q.; Du, C.-L.; Zhang, J.-X.; Wang, J.-Q.; Li, M.; Qi, W.-Y. (2018). Backfill support's backfill and operation properties and evaluation, Journal of Central South University, Vol. 25, No. 6, 1524-1534, doi:10.1007/s11771-018-3845-1

[5] Zhang, J. X.; Zhang, Q.; Spearing, A. J. S. S.; Miao, X. X.; Guo, S.; Sun, Q. (2017). Green coal mining technique integrating mining-dressing-gas draining-backfilling-mining, International Journal of Mining Science and Technology, Vol. 27, No. 1, 17-27, doi:10.1016/ j.ijmst.2016.11.014

[6] Meng, Z. S.; Zeng, Q. L.; Gao, K. D.; Kong, S.; Liu, P.; Wan, L. R. (2018). Failure analysis of super-large mining height powered support, Engineering Failure Analysis, Vol. 92, 378-391, doi:10.1016/j.engfailanal.2018.04.011

[7] Meng, Z. S.; Zeng, Q. L.; Wan, L. R.; Wang, C. L. (2018). Supporting performance and canopy adaptability of shield support, Journal of China Coal Society, Vol. 43, No. 4, 1162-1170, doi:10.13225/j.cnki.jccs.2017.1403

[8] Ge, X.; Xie, J. C.; Wang, X. W.; Liu, Y.; Shi, H. B. (2020). A virtual adjustment method and experimental study of the support attitude of hydraulic support groups in propulsion state, Measurement, Vol. 158, Paper 107743, 13 pages, doi:10.1016/j.measurement.2020.107743 
[9] Xie, J. C.; Wang, X. W.; Yang, Z. J.; Hao, S. Q. (2019). Attitude-aware method for hydraulic support groups in a virtual reality environment, Proceedings of the Institution of Mechanical Engineers, Part C: Journal of Mechanical Engineering Science, Vol. 233, No. 14, 4805-4818, doi:10.1177/0954406219838574

[10] Zhang, Y.; Zhang, H. Y.; Gao, K. D.; Xu, W. B.; Zeng, Q. L. (2019). New method and experiment for detecting relative position and posture of the hydraulic support, IEEE Access, Vol. 7, 181842-181854, doi:10.1109/ACCESS.2019.2958981

[11] Yang, X. J.; Wang, R. F.; Wang, H. F.; Yang, Y. K. (2020). A novel method for measuring pose of hydraulic supports relative to inspection robot using LiDAR, Measurement, Vol. 154, Paper 107452, 11 pages, doi:10.1016/j.measurement.2019.107452

[12] Wang, X. W.; Xie, J. C.; Shao, S. Q.; Li, Y. L.; Yang, Z. J.; Ren, F.; Tsing, Q. B. (2020). Key technologies of real-time virtual monitoring method for an intelligent fully mechanized coalmining face, Journal of China Coal Society, Vol. 45, No. 6, 1984-1996, doi:10.13225/j.cnki.jccs.zn20.0342

[13] Liang, M. F.; Fang, X. Q.; Li, S.; Wu, G.; Ma, M.; Zhang, Y. G. (2019). A fiber Bragg grating tilt sensor for posture monitoring of hydraulic supports in coal mine working face, Measurement, Vol. 138, 305-313, doi:10.1016/j.measurement.2019.02.060

[14] Cui, K. (2019). Analysis of supporting condition monitoring system of hydraulic support based on multi-sensor correction, Mechanical Management and Development, Vol. 10, No. 10, 152-153, doi:10.16525/j.cnki.cn14-1134/th.2019.10.062

[15] Asl, H. G.; Han, K. Y. (2019). Investigation on the influence of parameter uncertainties in the position tracking of robot manipulators, Tehnicki glasnik - Technical Journal, Vol. 13, No. 1, 1624, doi:10.31803/tg-20180921100632

[16] Mezyk, A.; Klein, W.; Fice, M.; Pawlak, M.; Basiura, K. (2016). Mechatronic model of continuous miner cutting drum driveline, Mechatronics, Vol. 37, 12-20, doi:10.1016/ j.mechatronics.2015.11.004

[17] Pan, D. L.; Gao, F.; Miao, Y. J.; Cao, R. (2015). Co-simulation research of a novel exoskeletonhuman robot system on humanoid gaits with fuzzy-PID/PID algorithms, Advances in Engineering Software, Vol. 79, 36-46, doi:10.1016/j.advengsoft.2014.09.005

[18] Kim, B. S.; Kim, T. G. (2019). Cooperation of simulation and data model for performance analysis of complex systems, International Journal of Simulation Modelling, Vol. 18, No. 4, 608619, doi:10.2507/IJSIMM18(4)491

[19] Nair, A. S.; Ezhilarasi, D. (2020). Performance analysis of super twisting sliding mode controller by ADAMS-MATLAB co-simulation in lower extremity exoskeleton, International Journal of Precision Engineering and Manufacturing - Green Technology, Vol. 7, No. 3, 743-754, doi:10.1007/s40684-020-00202-w

[20] Wen, S.-H.; Zheng, W.; Jia, S.-D.; Ji, Z.-X.; Hao, P.-C.; Lam, H.-K. (2020). Unactuated force control of 5-DOF parallel robot based on fuzzy PI, International Journal of Control, Automation and Systems, Vol. 18, No. 6, 1629-1641, doi:10.1007/s12555-018-0579-7

[21] Yang, Y.; Mi, Y. Q.; Qin, D. T.; Yuan, A. H.; Li, G. W. (2019). Analysis of the characteristics of electromechanical-hydraulic model of multi-source drive/transmission system based on periodic excitation, Advances in Mechanical Engineering, Vol. 11, No. 1, 1-15, doi: $10.1177 / 1687814018817128$

[22] Liu, G. J.; Zhou, Z. C.; Qian, X.; Wu, X. F.; Pang, W. H. (2016). Multidisciplinary design optimization of a swash-plate axial piston pump, Applied Sciences, Vol. 6, No. 12, Paper 399, 14 pages, doi:10.3390/app6120399

[23] Kutuk, M. E.; Dulger, L. C. (2018). Hybrid seven-bar press mechanism: link optimization and kinetostatic analysis, Tehnicki glasnik - Technical Journal, Vol. 12, No. 3, 181-187, doi: $10.31803 / \operatorname{tg}-20180203202102$

[24] Yang, Z. K.; Sun, Z. Y.; Jiang, S. B.; Mao, Q. H.; Liu, P.; Xu, C. Z. (2020). Structure analysis on impact-mechanical properties of ultra-high hydraulic support, International Journal of Simulation Modelling, Vol. 19, No. 1, 17-28, doi:10.2507/IJSIMM19-1-498

[25] Zeng, Q.-L.; Meng, Z.-S.; Wan, L.-R.; Wang, C.-L. (2018). Analysis on force transmission characteristics of two-legged shield support under impact loading, Shock and Vibration, Vol. 2018, Paper 3854684, 10 pages, doi:10.1155/2018/3854684 